\title{
Recurrence of Tumor Flare Reaction in a Chronic Lymphocytic Leukemia Patient During Chlorambucil- Rituximab Treatment 6 Years After Lenalidomide
}

\author{
Neno Živković ${ }^{1}$, Koraljka Gjadrov², Klara Dubravčić ${ }^{3}$, Sandra Bašić-Kinda ${ }^{4}$ and Igor Aurer ${ }^{1,4 *}$ \\ ${ }^{1}$ Medical School, University of Zagreb, Croatia \\ ${ }^{2}$ Department of Pathology and Cytology, University Hospital Centre Zagreb, Croatia \\ ${ }^{3}$ Department of Laboratory Diagnostics, University Hospital Centre Zagreb, Croatia \\ ${ }^{4}$ Division of Hematology, Department of Internal Medicine, University Hospital Centre Zagreb, Croatia
}

\begin{abstract}
In chronic lymphocytic leukemia, tumor flare reactions, characterized by sudden increase in tumor lesions caused by host immune effector cell infiltration, have been described exclusively after treatment with immunomodulators, most frequently lenalidomide. Here we describe a patient who had recurrence of tumor flare 6 years after the initial event, during treatment with rituximab and chemotherapy. This case report suggests that lenalidomide causes a long-lasting effect on lymph node microenvironment and host T cells.
\end{abstract}

KEYWORDS: Tumor flare reaction; Chronic lymphocytic leukemia; Rituximab; Lenalidomide

ABBREVIATIONS: CLL: Chronic Lymphocytic Leukemia; TRF: Tumor Flare Reaction

\section{INTRODUCTION}

Tumor flare reaction (TRF) is an increase in tumor lesion size, caused by sudden and massive infiltration of tumor by host effector cells, easily mistaken for disease progression [1]. In hematological diseases it has been most frequently described during treatment with immunomodulatory drugs lenalidomide and thalidomide [2-4]. Pathophysiology of TRF is still poorly understood. It is hypothesized that immunomodulatory drugs activate the host immune system's T-cell mediated immune response. Treatment of TRF is symptomatic with non-steroidal anti-inflammatory drugs and corticosteroids. Due to similarities with tumor progression, careful evaluation is needed, but once tumor progression is excluded, same anti-tumor therapy may be continued. In chronic lymphocytic leukemia (CLL)
TRF presents with enlarged painful tender lymph nodes with rash and low-grade fever usually occurring in lymph nodes primary enlarged due to tumor infiltration. We present a case report of a patient with CLL who developed two tumor flare reactions in the same lymph node 6 years apart, the first after lenalidomide and the second after rituximab treatment.

\section{CASE PRESENTATION}

A 73-year-old Caucasian female with progressive CLL was started on lenalidomide $2.5 \mathrm{mg}$ daily. After 2 days, fever and painful massive swelling of cervical lymph nodes and pharyngeal Waldeyer's ring lymphatic tissue occurred. Fine needle aspirate of
Quick Response Code:

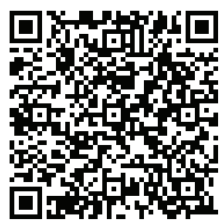

Address for correspondence: Igor Aurer, Division of Hematology, Department of Internal Medicine, University Hospital Centre Zagreb, Croatia

Received: July 05, 2021 Published: July 28, 2021

How to cite this article: Zivkovic N, Gladrov K, Dubravcic K, Basic-Kinda S, Aurer I. Recurrence of Tumor Flare Reaction in a Chronic Lymphocytic Leukemia Patient During ChlorambucilRituximab Treatment 6 Years After Lenalidomide. 2021- 3(4) OAJBS.ID.000310. DOI: 10.38125/ OAJBS.000310 
affected lymph nodes confirmed a tumor flare reaction. Ibuprofen did not sufficiently alleviate the symptoms, the patient received steroids, lenalidomide was stopped and treatment with rituximab and chlorambucil initiated, resulting in remission. After 5.5 years progressive lymphadenopathy and splenomegaly occurred. Evaluation confirmed CLL progression. Due to long first remission, a decision to repeat treatment with chlorambucil and rituximab was made. She received her first dose of rituximab 70 months after the initial tumor flare reaction. Three weeks later, while on prophylactic acyclovir, she developed fever, painful swelling of lymph nodes in cervical regions 1 and 2, edema of pharyngeal mucosa and aftous stomatitis, consistent with a tumor flare reaction. At that time her neutrophil count was $2,7 \times 10^{\wedge 9} / \mathrm{l}$, and her lymphocyte count dropped from pretreatment 14.3 to $0.5 \times 10^{9} / \mathrm{l}$, with less than $1 \%$ being B-cells. No pathogenic bacteria nor fungi were isolated from swabs. Antimicrobial treatment was ineffective, but the patient promptly responded to steroids. Treatment with rituximab and chlorambucil was continued and a $2^{\text {nd }}$ remission achieved uneventfully.

\section{DISCUSSION}

We performed a search of PubMed and found that all reported tumor flare reactions in CLL patients occurred during treatment with lenalidomide. No reports were related to rituximab and chlorambucil treatment. We hypothesize that this tumor flare reaction is related to previous exposure to lenalidomide. The study of Strati et al shows that clinical effects of lenalidomide persist long after its discontinuation [5], possibly due to interactions between CLL cells and the immune microenvironment. Lenalidomide activates the host immune system by activating CD8+ cytotoxic $\mathrm{T}$ lymphocytes and downregulating regulatory $\mathrm{T}$-cell supressor function [6,7]. It also enhances antitumor immune responses mediated by NK and CD4+ T cells [8]. Lenalidomide promotes CD154 expression on CLL cells and enhances production of antibodies by normal B cells through a PI3-kinase-dependent pathway [9]. These effects on the host immune system are long-lasting. Our patient developed her second tumor flare reaction in the same lymphatic region 71 months after the first one, consistent with a possible long lasting lenalidomide effect on lymph node microenvironment and host $\mathrm{T}$ cells triggered by tumor cell destruction caused by immunochemotherapy.

\section{CONCLUSION}

In conclusion, this case report suggests that the immune effects of lenalidomide causing TFR might be permanent and be triggered by local tumor cell destruction by other agents besides immunomodulators.

\section{ACKNOWLEDGEMENT}

Supported by grant 1120002 from the University of Zagreb.

\section{CONFLICT OF INTEREST}

KD, SBK and IA received support and/or honoraria from Roche. SBK and IA received support and honoraria from Sandoz, Oktalpharma and Celgene.

\section{REFERENCES}

1. Taleb $A B$ (2019) Tumour flare reaction in cancer treatments: a comprehensive literature review. Anti-Cancer Drugs 30: 954-958.

2. Chanan-Khan A, Miller KC, Takeshita K, Koryzna A, Donohue K, et al. (2005) Results of a phase 1 clinical trial of thalidomide in combination with fludarabine as initial therapy for patients with treatment-requiring chronic lymphocytic leukemia (CLL). Blood 106: 3348-3352.

3. Chanan-Khan AA, Chitta K, Ersing N, Paulus A, Masood A, et al. (2011) Biological effects and clinical significance of lenalidomide-induced tumour flare reaction in patients with chronic lymphocytic leukaemia: in vivo evidence of immune activation and antitumour response. $\mathrm{Br} \mathrm{J}$ Haematol 155: 457-467.

4. Andritsos LA, Johnson AJ, Lozanski G, Blum W, Kefauver C, et al. (2008) Higher doses of lenalidomide are associated with unacceptable toxicity including life-threatening tumor flare in patients with chronic lymphocytic leukemia. J Clin Oncol 26: 2519-2525.

5. Strati P, Ferrajoli A, Wierda WG, Jain N, Thompson PA, et al. (2018) Sustained long-lasting responses after lenalidomide discontinuation in patients with chronic lymphocytic leukemia. Leukemia 32: 2278-2281.

6. Ramsay AG, Johnson AJ, Lee AM, Gorgun G, Le Dieu R, et al. (2008) Chronic lymphocytic leukemia $\mathrm{T}$ cells show impaired immunological synapse formation that can be reversed with an immunomodulating drug. J Clin Invest 118: 2427-2437.

7. Ramsay AG, Evans R, Kiaii S, Svensson L, Hogg N, et al. (2013) Chronic lymphocytic leukemia cells induce defective LFA-1-directed T-cell motility by altering Rho GTPase signaling that is reversible with lenalidomide. Blood 121: 2704-2714.

8. Acebes-Huerta A, Huergo-Zapico L, Gonzalez-Rodriguez AP, Fernandez-Guizan A, Payer AR, et al. (2014) Lenalidomide induces immunomodulation in chronic lymphocytic leukemia and enhances antitumor immune responses mediated by NK and CD 4 T cells. Biomed Res Int 2014: 265840.

9. Lapalombella R, Andritsos L, Liu Q May SE, Browning R, et al. (2010) Lenalidomide treatment promotes CD154 expression on CLL cells and enhances production of antibodies by normal B cells through a PI3kinase-dependent pathway. Blood 115: 2619-2629. 